Revisión

\title{
Guías Clínicas en Sarcoma de Partes Blandas
}

\author{
Consenso del Grupo Español de Investigación en Sarcomas (GEIS)
}

Los sarcomas de partes blandas (SPB) son un grupo de tumores poco frecuentes, pues suponen menos del $1 \%$ de todas las neoplasias. Están constituidos por más de 20 subtipos histológicos diferentes que se agrupan por tener un comportamiento biológico semejante, con el propósito de estudiar sus factores pronósticos y analizar los resultados terapéuticos. En este documento se definen y establecen distintos aspectos relacionados con su diagnóstico y tratamiento a partir de las conclusiones acordadas en la Reunión del Grupo GEIS celebrada en Bayona el 26-27 de Marzo de 2004. El documento proporciona una guía de buena práctica clínica para el tratamiento de pacientes con enfermedad localizada o metastásica, y propone normas para el desarrollo futuro de fármacos en enfermos con un SPB.

En un futuro podría valorarse el grado de cumplimiento de estas normas por los distintos Centros, y valorar su impacto en los resultados del tratamiento. No deben considerarse como un texto definitivo, sino que deberá mejorarse a partir de la experiencia acumulada con su utilización.

\section{Enfermedad limitada}

\subsection{Estadificación y biopsia}

El 60\% de los pacientes con SPB debuta con una tumoración en una extremidad. En un 20\% de casos el tumor primario tiene localización retroperitoneal. El aumento de tamaño de una lesión ya conocida o la presencia de una masa de tamaño igual o superior a $5 \mathrm{~cm}$ en una extremidad son criterios de sospecha clínica.

La resonancia magnética (RM), para evaluar el tumor primario, y la radiología simple de tórax, para descartar diseminación pulmonar, se consideran pruebas de estadificación estándar para tumores primarios de una extremidad, de la cabeza o el cuello. En los retroperitoneales, la tomografía computerizada (TC) abdominal y la radiología simple de tórax se consideran pruebas estándar, mientras que en los tumores originados en el tórax, la TC torácica y de hemiabdomen superior sería el proceder adecuado.

En lo que respecta a los SPB localizados en las extremidades y en el tronco superficial, y contrariamente a lo que sucede en España, numerosos países europeos han desarrollado estrategias para el envío de pacientes con un diagnóstico de sospecha a centros de referencia (CR). Los centros de referencia deberían disponer, como mínimo, de un equipo especializado en cirugía de SPB que integre las distintas disciplinas necesarias para el manejo de estos enfermos- radioterapia, anatomía patológica, radiología y oncología médica, por lo menos- que diagnostique y trate un número de pacientes, entre 15-20 pacientes al año, que le permita alcanzar una experiencia suficiente.

En España, de acuerdo con los datos del Registro GEIS de SPB, la biopsia escisional (enucleación) se lleva a cabo en un $49 \%$ de los tumores con un tamaño igual o superior a $5 \mathrm{~cm}$ localizados en extremidades, la cabeza o el tronco. Esto indica que, en al menos la mitad de los casos, se aborda la lesión sin un diagnóstico de sospecha, o con un desconocimiento del proceder inicial correcto, interfiriendo con las actuaciones posteriores. El porcentaje de biopsias escisionales llevadas a cabo por el Grupo Escandinavo de Sarcomas (SSG), grupo que sigue unas pautas muy definidas de actuación, se sitúa por de- 
bajo del 20\%. Para mejorar esta situación, sólo deberían abordarse en un centro secundario aquellos pacientes con un tumor superficial de un tamaño inferior a $3 \mathrm{~cm}$, intentando en la resección obtener márgenes negativos superiores a $1 \mathrm{~cm}$, derivando posteriormente al paciente a un CR para la evaluación de tratamiento complementario. El resto de pacientes debería ser remitido directamente. Deberían ponerse en marcha mecanismos que aseguren el cumplimiento de estos criterios.

El tratamiento de un SPB se basa en un diagnóstico histológico correcto obtenido mediante una técnica que no dificulte actuaciones posteriores, realizada preferiblemente por el equipo que va a tratar al paciente. Con este propósito, la biopsia con tru-cut resulta, cuando menos, tan válida como la biopsia incisional, y causa menos complicaciones locales. El anatomopatólogo debe proporcionar al clínico el tipo histológico y el grado de malignidad tumoral de la lesión. La punción-aspiración con aguja fina (PAAF) podría ser un método eficaz para orientar el diagnóstico en centros con un radiólogo y un citólogo expertos. La PAAF estaría indicada en pacientes con enfermedad metastásica o en los que la estrategia quirúrgica posterior no vaya a cambiar aunque, lógicamente, no puede establecer el grado de malignidad y, en ocasiones, tampoco el tipo histológico concreto.

\subsection{Tratamiento quirúrgico}

La cirugía amplia (con márgenes) o la cirugía radical (amputación o compartimental) se consideran tratamientos adecuados en los SPB de extremidades, siempre que proporcionen unos márgenes, tanto laterales como profundos, libres de enfermedad microscópica ${ }^{1}$. Estos márgenes deberían ser de al menos $1 \mathrm{~cm}$ en todas las direcciones. No es posible practicar una cirugía que cumpla estos requisitos en regiones como la zona inguinal, la fosa poplítea, el pié, la mano, el codo, o en la mayoría de las regiones del tronco.

En el retroperitoneo tampoco es posible practicar una cirugía adecuada y en centros con experiencia sólo se consigue una resección macroscópicamente completa del tumor en el $60 \%$ de los casos.

El informe de Anatomía Patológica definitivo debe incluir, en todos los casos, el tipo histológico, el grado histológico de malignidad, el tamaño del tumor, y la distancia en milímetros del tumor a todos los bordes de resección, y la eventual afectación ganglionar, muy improbable en SPB. La pieza quirúrgica, por tanto, debe llegar al patólogo convenientemente marcada. Para asignar el grado de malignidad es recomendable aplicar los criterios de la Federación Francesa de Centros contra el Cáncer (FNCLCC). El índice de Ki-67 no está validado como un parámetro para asignar el grado de malignidad.

\subsection{Tratamiento complementario}

En lesiones originadas en una extremidad deberá administrarse radioterapia externa (RT) postoperatoria en las siguientes situaciones ${ }^{2}$ :

1. después de la extirpación de una recidiva local, con independencia del tamaño de la lesión o de su distancia a los bordes

2. en casos sometidos a una re-escisión después de una intervención quirúrgica no planificada con independencia de que exista o no tumor en la pieza quirúrgica

3. en tumores primarios sometidos a una cirugía amplia (márgenes negativos $>10 \mathrm{~mm}$ ), con un tamaño igual o superior a $5 \mathrm{~cm}$ y de alto grado.

4. en tumores con márgenes microscópicos positivos (R1) o unos bordes negativos $<10 \mathrm{~mm}$, en los que la reintervención no pueda plantearse por cuestiones técnicas (infiltración de estructuras vitales adyacentes) o por negativa del paciente.

La braquiterapia complementaria o adyuvante tendría estas mismas indicaciones, aunque su uso estaría restringido a centros con una experiencia acreditada. Su utilidad en lesiones de bajo grado es controvertida ${ }^{3}$.

Podría omitirse la RT complementaria en los pacientes con lesiones de alto grado y de un diámetro $<5 \mathrm{~cm}$, o en aquéllos con lesiones de bajo grado de cualquier tamaño, tratados mediante una cirugía amplia con márgenes negativos $>10 \mathrm{~mm}$, siempre que no hayan sido sometidos a una escisión (enucleación) previa ${ }^{4}$. En los tumores extirpados mediante una cirugía compartimental adecuada tampoco está indicada la RT adyuvante.

La radioterapia debería iniciarse en los 2 meses siguientes a la cirugía y la dosis mínima establecida es de $60 \mathrm{~Gy}$, con un fraccionamiento diario de 1.8 
Gy. No se ha demostrado la eficacia de la RT complementaria en los tumores retroperitoneales.

El pronóstico de los pacientes con una recidiva local tratados aplicando los criterios anteriores es semejante al del tratamiento de una lesión primaria.

Se mantienen las recomendaciones que ya existen sobre la quimioterapia adyuvante. Podría utilizarse en pacientes con un SPB de localización profunda, con un tamaño igual o superior a $5 \mathrm{~cm}$, y de alto grado de malignidad, que presentan un riesgo de recidiva del $60 \%$ aproximadamente ${ }^{5,6}$. En todo caso esta decisión debe acordarse con cada paciente, dado que la administración de este tratamiento sólo aumenta la supervivencia global en un $7 \%$ en pacientes con una lesión de extremidades, y que los esquemas con doxorrubicina y dosis altas de ifosfamida que deberían administrarse no están exentos de toxicidad. Quizá sus indicaciones puedan definirse mejor a partir de los resultados de los estudios, actualmente en curso, promovidos por el Grupo de Sarcomas Italiano y por la Organización Europea para la Investigación y Tratamiento del Cáncer.

\subsection{Seguimiento}

La práctica de una radiografía simple de tórax y la evaluación clínica del lecho quirúrgico sería una estrategia correcta de seguimiento en los tumores resecados en la extremidad. La Rx de tórax se realizará cada 2-3 meses, durante los 2 primeros años y, posteriormente, cada 4-6 meses hasta los 5 años. La TC de tórax confirmaría los hallazgos de la radiografía de tórax y ayudaría a evaluar las opciones quirúrgicas del paciente. En caso de sospecha clínica de recidiva local, debería realizarse una RM o una ecografía.

En el seguimiento de los tumores retroperitoneales de alto grado, el TC abdominal repetido cada 3 meses durante los 2 primeros años, y luego cada 6 meses hasta los 5 años, puede ser una estrategia adecuada. En lesiones de bajo grado el estudio podría repetirse cada 6 meses.

\section{Enfermedad metastásica}

\subsection{Estadificación}

Se consideran pruebas de estadificación correctas para pacientes con enfermedad diseminada la radio- grafía simple de tórax y la TC abdominal. Las lesiones pulmonares son las más frecuentes, siendo la única localización metastásica en el $40 \%$ de los casos. Si la enfermedad sólo afecta a los pulmones y es técnicamente resecable ( $<5$ nódulos) debería realizarse una TC helicoidal de tórax con cortes cada 5 $\mathrm{mm}$ para descartar la presencia de lesiones de tamaño < $10 \mathrm{~mm}$. La RM o la gammagrafia ósea podrían estar indicadas en casos concretos.

\subsection{Factores pronósticos}

La heterogeneidad de la muestra constituye una de las principales deficiencias de los ensayos clínicos realizados en pacientes con un SPB diseminado, en parte determinada por la baja prevalencia de esta patología. La agrupación de los enfermos en función del tipo histológico no es viable excepto en proyectos en los que participen distintos Grupos Cooperativos.

Los estudios de quimioterapia deberían contemplar, por una parte, el tipo de diseminación y, por otra, el estado general de los enfermos, aspectos que influyen en los resultados esperables (Fig. 1). Actualmente, los pacientes con una metástasis pulmonar única se incluyen junto a los pacientes con metástasis hepáticas, ganglionares u óseas, cuando su pronóstico es muy diferente. De la misma manera, también debería establecerse "a priori" si las lesiones son o no resecables. En todos los ensayos clínicos hay un porcentaje de pacientes (fundamentalmente con metástasis pulmonares) que son rescatados con cirugía, pero en ningún trabajo se especifica su situación de partida. Algunos de estos pacientes serían muy posiblemente candidatos a una cirugía de entrada, y no deberían haber sido incluidos en el estudio; alternativamente, si son incluidos, deberían estratificarse teniendo en cuenta su mejor pronóstico. En pacientes con afectación pulmonar exclusiva sometidos a cirugía de rescate, el intervalo libre de enfermedad, la edad del paciente, el número de nódulos y la resección completa de las mismos, son factores pronósticos de supervivencia favorables ${ }^{7-9}$.

El estado general, la presencia de metástasis hepáticas, un alto grado de malignidad y la edad avanzada son factores pronósticos desfavorables respecto a la supervivencia, mientras que un intervalo libre prolongado es un factor favorable ${ }^{10}$. 


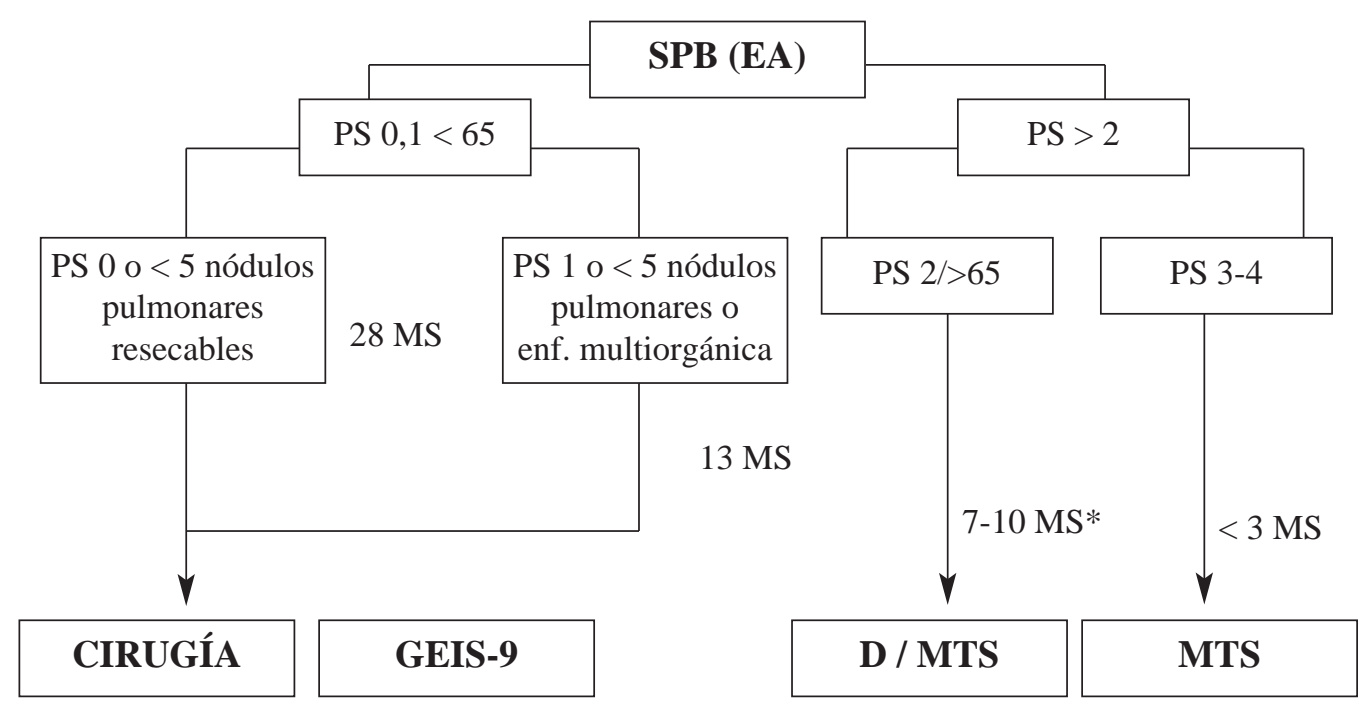

SPB: Sarcoma Partes Blandas; EA: Enfermedad Avanzada; MTS: Mejor Tratamiento de Soporte; D: Doxorubicina.

Figura 1. Algoritmo pronóstico en SPB con enfermedad avanzada.

\subsection{Tratamiento}

Cuando es posible, la resección completa de las metástasis es el tratamiento de elección, aunque la experiencia más contrastada se ha obtenido a partir de la exéresis de lesiones pulmonares.

La quimioterapia es el tratamiento de elección en paciente con enfermedad multiorgánica no cantidatos a un rescate quirúrgico. Hay dos grupos de fármacos con actividad en el tratamiento de un SPB metastásico, las antraciclinas (doxorrubicina y 4epi-doxorrubicina), con una actividad como primera línea terapéutica confirmada por múltiples estudios que varía entre el 9 y el $23 \%{ }^{11,12}$ y las oxazofosforinas (ciclofosfamida e ifosfamida). La ifosfamida administrada a dosis superiores a $10 \mathrm{~g} / \mathrm{m}^{2}$ (HD-IFOS) proporciona, como agente único, hasta un $40 \%$ de respuestas objetivas ${ }^{13}$. Si bien la doxorrubicina o la 4-epidoxorrubicina son bien toleradas por la mayoría de los pacientes, la HD-IFOS y sus combinaciones con una antraciclina provocan una toxicidad elevada, y no se ha demostrado que sean superiores, en términos de mejora en la supervivencia $y$ calidad de vida $^{14-17}$. El tratamiento estándar de primera línea en todos los pacientes con buen estado general (de 0 a 2 en la escala de la OMS) es la doxorrubicina a dosis de $75 \mathrm{mg} / \mathrm{m}^{2}$ repetida cada 21 días.

Los esquemas de poliquimioterapia deberían uti- lizarse únicamente en el contexto de un ensayo clínico (18-19). Deberían de excluirse de estos ensayos los pacientes con estado general $>1$ y aquellos de edad > de 65 años, dado que la toxicidad en esta población es excesiva. Estos pacientes podrían constituir el grupo control de ensayos clínicos comparativos que pretendan establecer los posibles beneficios de un nuevo fármaco.

\section{Metodología para el desarrollo de nuevos fármacos en SPB}

Eventualmente, la mayoría de los pacientes progresan a los tratamientos de primera línea y pueden ser candidatos a un segundo o tercer intento terapéutico con quimioterapia. El impacto de estos tratamientos en la supervivencia y en la calidad de vida de los pacientes no está demostrado. La actividad de la ifosfamida y del DTIC en pacientes previamente tratados oscila entre el 8 y el $24 \%^{20-22}$, y estos fármacos se consideran activos como segunda línea terapéutica.

Ninguno de los fármacos desarrollados en los últimos 10 años ha mostrado una actividad consistente y reproducible frente a los SPB. La gemcitabina, la temozolamida o la ectainascidina (ET-743) han mostrado una cierta actividad en segunda o tercera 
TABLA I

Estudios con nuevos fármacos en segunda línea en SPB

\begin{tabular}{|c|c|c|c|c|c|c|c|}
\hline Autor & No. & Fármaco & $\begin{array}{c}P S \\
(>1)\end{array}$ & $\begin{array}{c}I L E \\
(<12)\end{array}$ & $\begin{array}{c}\text { Evaluación } \\
\text { (semanas) }\end{array}$ & $\begin{array}{l}R O \\
(\%)\end{array}$ & $\begin{array}{c}\text { MTTP } \\
\text { semanas }\end{array}$ \\
\hline Patel* & 39 & $\mathbf{G}$ & 7 & - & 8 & 18 & 12 \\
\hline Svancarova & 32 & G & 0 & - & 6 & 3 & 6 \\
\hline Okuno & 30 & $\mathrm{G}$ & 14 & - & - & 3 & 8 \\
\hline Woll & 31 & $\mathrm{~T}$ & 0 & - & - & 3 & 8 \\
\hline Yovine & 56 & ET & 0 & 21 & 6 & 4 & 8 \\
\hline G-Carbonero & 36 & ET & 0 & 22 & 6 & 6 & 8 \\
\hline
\end{tabular}

*7\% de los pacientes tratados en primera línea

No.: número de pacientes. PS: estado general (ECOG). ILE: intervalo libre de enfermedad (meses). RO: respuestas objetivas. MTTP: mediana de tiempo hasta la progresión. G: gemcitabina. T: temozolamida. ET: ectainascidina-743.

línea, con un índice de respuestas objetivas que varía entre el 3 y el $18 \%{ }^{23-28}$ (Tabla I). Ninguno de estos fármacos ha sido comparado con la doxorrubicina en primera línea, y un estudio de este tipo realizado para confirmar la eficacia del taxotere arrojó un resultado negativo ${ }^{29,30}$, lo que obliga a reflexionar sobre la metodología utilizada en el desarrollo de nuevos fármacos en SPB.

Recientemente se ha propuesto como parámetro principal de valoración de eficacia de nuevos fármacos en pacientes con un SPB el porcentaje de pacientes libres de progresión (PFR) a los 3 meses de su inclusión en el estudio ${ }^{31}$. El PFR podría ser útil no sólo para valorar la eficacia de los fármacos citotóxicos convencionales, sino también, de los nuevos fármacos citostáticos. Después de la administración en segunda línea de medicamentos considerados activos, como la dacarbazina y la ifosfamida, el PFR a los 3 y 6 meses es del $39 \%$ y $14 \%$, con cifras del 21 y $8 \%$, respectivamente, para los fármacos inactivos. Un incremento sustancial de este parámetro indicaría probablemente una actividad biológica interesante. Nosotros proponemos un PFR del 60\% a los 3 meses como un claro índice de actividad para un nuevo fármaco. En ensayos comparativos que utilizaran este parámetro debería incluirse un análisis intermedio del PFR que permitiera la suspensión del estudio si es improbable que se alcancen los objetivos propuestos ${ }^{32}$.

En los nuevos estudios se debería comparar, al azar, una asociación del fármaco investigacional con el principio activo convencional, o bien éste último con el nuevo fármaco ${ }^{33}$. La respuesta objetiva, medida con criterios aceptados (RECIST) ${ }^{34}$, sería un objetivo secundario, y deberían aplicarse unos criterios estrictos para determinar el momento de la progresión, con independencia de que el paciente hubiera abandonado o no el estudio. Los enfermos, como hemos comentado anteriormente, deberían estratificarse en función del estado general ( 0 ó 1 frente a 2) y del intervalo libre de enfermedad ( $>12$ meses frente a $<12$ meses).

A la par que se realiza el ensayo clínico, es necesario desarrollar estudios translacionales (farmacogenómicos y farmacodinámicos) que permitan profundizar en el conocimiento de los SPB.

Correspondencia:

Dr. J. Maurel Hospital Clinic Barcelona 


\section{Bibliografía}

1. Rosemberg SA, Tepper J, Glatstein E, et al. The treatment of soft tissue sarcomas of the extremities. Ann Surg 1982; 196: 305-315.

2. Yang JC, Chang AE, Baker AR, et al. Randomized prospective study of the benefit of adjuvant radiation therapy in the treatment of soft tissue sarcomas of the extremity. J Clin Oncol 1998; 16: 197-203.

3. Pisters PW, Harrison LB, Leung DH, et al. Long-term results of a prospective randomized trial of adjuvant brachytherapy in soft tissue sarcoma. J Clin Oncol 1996; 14: 859868.

4. Rydholm A, Gustafson P, Rooser B, et al. Limb-sparing surgery without radiotherapy based on anatomic location of soft tissue sarcoma. J Clin Oncol 1991; 9: 1757-1765.

5. Adjuvant chemotherapy for localised resectable soft-tissue sarcoma of adults: Meta-analysis of individual data. Sarcoma Meta-Analysis collaboration. Lancet 1997; 350: 16471654.

6. Frustaci S, Gherlinzoni F, De Paoli A, et al. Adjuvant chemotherapy for adult soft tissue sarcomas of the extremities and girdles: results of the italian randomized cooperative trial. J Clin Oncol 2001; 19: 1238-1247.

7. Van Geel R, Pastorino U, Jauch KW et al. Surgical treatment of lung metastases. The EORTC STBSG study of 255 patients. Cancer 1996; 77: 675-682.

8. Billingsley KG, Lewis JJ, Leung DHY, et al. Multifactorial analysis of the survival or patients with distant metastasis arising from primary extremity sarcomas. Cancer 1999; 85: 389-395.

9. Billingsley KG, Burt ME, Jara E, et al. Pulmonary metastases from soft tissue sarcoma. Analysis of patterns of disease and postmetastases survival, Ann Surg 1999; 229: 602-612.

10. Van Glabbeke M, Van Oosterom AT, Oosterhuis JW, et al. Prognostic factors for the outcome of chemotherapy in advanced soft tissue sarcoma: an analysis of 2,185 patients treated with anthracyclines-containing first-line regimensA European Organization for Research and Treatment of Cancer Soft Tissue and Bone Sarcoma Group Study. J Clin Oncol 1999; 17: 150-157.

11. Mouridsen HT, Bastholt L, Somers R, et al. Adriamycin versus epirubicin in advanced soft tissue sarcomas. A randomized phase II/phase III study of the EORTC Soft Tissue and Bone Sarcoma Group. Eur.J. Cancer 1987; 23: 1477-1483.

12. Lorigan PC, Verweij J, Papai Z, et al. Randomised phase III trial of two investigational schedules of ifosfamide versus standad dose doxorubicin in patients with advanced or metastatic soft tissue sarcoma. Proc Ann met Amer Soc Clin Onc 2002; 20: 405.

13. Buesa JM, López-Pousa A, Martín J y cols. Phase II trial of first-line high-dose ifofamide in advanced soft tissue sarcomas of the adult: a study of the Spanish Group for Research on Sarcomas. Ann Oncol 1998; 9: 871-876.
14. Frustaci S, Buonadonna A, Galligioni E, et al. Increasing 4-epidoxorubicin and fixed ifosfamide doses plus granulocyte-macrophage colony-stimulating factor in advanced soft tissue sarcomas: a pilot study. J Clin Oncol 1997; 15: 1418-1426.

15. Reichardt P, Tilgner J, Hohenberger P, Dörken B. Dose-intensive chemotherapy with ifosfamide, epirubicin, and filgastrim for adult patients with metastatic or locally advanced soft tissue sarcoma: a phase II study. J Clin Oncol 1998; 16; 1438-1443.

16. De Pas T, De Braud F, Orlando L, et al. High-dose ifosfamide plus adriamycin in the treatment of adult advanced soft tissue sarcomas: it is feasible?. Ann Oncol 1998; 9; 917-919.

17. Maurel J, Fra J, López-Pousa A, et al. Sequential dosedense doxorubicin and ifosfamide for advanced soft tissue sarcomas. A phase II trial by the Spanish Group for Research on Sarcomas (GEIS). Cancer 2004; 100: 1498-506.

18. Edmonson JH, Ryan LM, Blum RG, et al. Randomized comparison of doxorubicin alone versus ifosfamide plus doxorubicin or mitomycin, doxorubicin and cisplatin against advanced soft tissue sarcomas. J Clin Oncol 1993; 11: 1269-1275.

19. Santoro A, Rouessé J, Steward W, et al. Doxorubicin versus CYVADIC versus doxorubicin plus ifosfamide in firstline treatment advanced soft tissue sarcomas: a randomized study of the European Organization for Research and Treatment of Cancer Soft Tissue and Bone Sarcoma Group. J Clin Oncol 1995; 13: 1537-1545.

20. Antman KH, Ryan L, Elias A, Sherman D, Grier E. Response to ifosfamide and mesna: 124 previously treated patients with metastatic or unresectable sarcoma. J Clin Oncol 1989; 7: 126- 131.

21. Le Cesne A, Antoine E, Spielman M, Le Chevalier T, et al. High-dose ifosfamide: circumvention of resistance to standard-dose ifosfamide in advanced soft tissue sarcomas. J Clin Oncol 1995; 13: 1600-1608.

22. Buesa JM, Mouridsen HT, Oosterom ATV et al. High-dose DTIC in advanced soft- tissue sarcomas in the adult. A phase II study of the EORTC soft tissue and bone sarcomas group. Ann Oncol 1991; 2: 307-309.

23. Patel SR, Gandhi V, Jenkins J et al. Phase II clinical investigation of gemcitabine in advanced soft tissue sarcomas and window evaluation of dose rate on gemcitabine triphosphate accumulation. J Clin Oncol 2001; 19: 3483-3489.

24. Svancarova L, Blay JY, Judson IR, et al. Gemcitabine in advanced adult soft-tissue sarcomas. A phase II study of the EORTC Soft Tissue and Bone Sarcoma Group. Eur J Cancer 2002; 38: 556-559.

25. Okuno S, Edmonson J, Mahoney M, et al. Phase II trial of gemcitabine in advanced sarcomas. Cancer 2002; 94: 3225-9.

26. Woll PJ, Judson I, Lee SM, et al. Temozolamide in adult patients with advanced soft tissue sarcoma: a phase II study of the EORTC Soft Tissue and Bone Sarcoma Group. Eur J Cancer 1999; 35: 410-412. 
27. Yovine A, Riofrio M, Blay JY, et al. Phase II study of ectainascidin-743 in advanced pretreated soft tissue sarcoma patients. J Clin Oncol 2004; 22: 890-899.

28. R Garcia-Carbonero, Supko JG, Manola J, et al. Phase II and pharmacokinetic study of ectainascidin-743 in patients with progressive sarcomas of soft tissues refractory to chemotherapy. J Clin Oncol 2004; 22:1480-1490.

29. Verweij J, Lee SM, Ruka W, Buesa J, Coleman R, Van Hoessel R, et al. Randomized phase II study of docetaxel versus doxorubicin in first- and second -line chemotherapy for locally advanced or metastatic soft tissue sarcomas in adults: A Study of the European Organization for Research and Treatment of Cancer Soft Tissue and Bone Sarcoma Group. J Clin Oncol 2000; 18: 2081-2086, 2000

30. Van Hoesel QG, Verweij J, Catimel G, et al. Phase II study with docetaxel (taxotere) in advanced soft tissue sarcomas in adults: a study of the European Organization for Research and Treatment of Cancer Soft Tissue and Bone Sarcoma Group. Ann Oncol 1994; 5: 539-542.

31. Van Glabekke M, Verweij J, Judson I, et al. Progressionfree rate as the principal end-point for phase II trials in soft-tissue sarcomas. Eur J Cancer 2002; 38: 543-549.

32. Dent S, Zee B, Dancey J, et al. Application of a new multinomial phase II stopping rule using response and early progression. J Clin Oncol 2001; 19: 785-791.

33. Van Glabbecke M, Steward W, Armand JP. Non-randomized phase II trials of drug combinations :often meaningless, sometimes misleading. Are there alternative strategies? Eur J Cancer 2002 ; 38: 635-638.

34. Therasse P, Arbuck SG, Eisenhauer EA, et al. New guidelines to evaluate the response to treatment in solid tumors. J Natl Cancer Inst 2000; 92: 205-216.

\section{Grupo Español de Investigación de Sarcomas (GEIS)}

Alija V, Alvarez E, Andres R, Antón LM, Aranda E, Balañá C, Barrajón E, Bellmunt J, Blanco R, Blasco A, Bover I, Buesa JM, Calvo E, Cano JM, Camps C, Carabantes F, Carles Galcerán J, Carrasco JA, Casado Echarren V, Casado Herráez A, Casinello J, Contreras JA, Cortes Funes H, Cruz Jurado J, Cruz Hernandez JJ, Cubedo R, Cubillo A, Cuevas JM, Da Silva R, de Juan A, de las Peñas R, Dinis J, Domínguez S, Dorta FJ, Escudero P, Fabregat X, Fra J, Gallego O, García Castro I, García del Muro X, García Girón C, García Gómez J, García Teijido P, Gómez Bernal A, Gómez España MA, Gómez Martín C, González Billalabeitia E, González Campora R, González A, González de Sande LM, Guillén C, Hitt R, Jara C, Juan O, Lainez N, Lasso R, Lavernia J, Lianes P, Llorente R, Llosa F, López Pousa A, López-Vega JM, López Vivanco G, Maestu I, Martín Algarra S, Martín Broto J, Martínez Trufero J, Massuti B, Maurel J, Meana A, Menendez MD, Molano F, Molins C, Montalar J, Morán T, Muñoz A, Pericay C, Piera JM, Poveda A, Puertas J, Roca JM, Rodríguez Salas N, Rubio Viqueira B, Rubió Casadevall J, Safont MJ, Sánchez Heras A, Santomeu L, Sevilla I, Soares M, Vadell C, Valentí V, Valladares M, Vázquez S, Vélez E, Vidal Y, Yubero A, Yuste A. 